\section{Urinary tract infection due to beta-lactams-resistant Staphylococcus warneri: A case report}

\section{Abstract}

Staphylococcus warneri, similarly to other coagulase-negatine Staphylococcus (CoNS), is a commensal of the skin, mucous membranes, nasal cavities and mouth of humans, found in $50 \%$ of the population. This species usually represents less than $1 \%$ of the total staphylococcal skin population. S. warneri can potentially cause severe infections usually in immunocompromised individuals, even more in combination with the presence of indwelling devices and/or implants. The key treatment for such infections is the removal of the device. Sometimes, even in the absence of a foreign body, Staphylococcus warneri may be implicated as the causative agent of an infection in immunocompetent patients. We report here a case of chronic urinary tract infection in adult healthy patient caused by S.warneri, its clinical significance and resistant to commonly used beta-lactams

Keywords: Staphylococcus warneri, urine infection, neurogenic bladder
Dimitra Dimitriadi', Christina Charitidou², Ekatherina Charvalos ${ }^{3}$

1 In Vitro Labs.

2 Private Microbiological Laboratory.

3 laso Hospital.

Corresponding author:

\section{Dimitra Dimitriadi}

”dimdimitriadi@hotmail.com

\section{Introduction}

Urinary tract infections (UTI) remain one of the most prevalent and frustrating morbidities for neurogenic bladder patients. Risk factors include urinary stasis, high bladder pressures, bladder stones, and catheter use. While classic symptoms of UTI include dysuria, increased frequency and urgency, neurogenic bladder patients present differently with increased spasticity, urinary incontinence, and vague pains. Escherichia coli is the most common isolated pathogen, followed by Klebsiella pneumonia, Enterococcus faecalis, Proteus mirabilisand Pseudomona saeruginosa [1].

Most clinical isolates of CoNS were mostly susceptible to penicillins few decades ago.

Here we present a rare case of urinary tract infection caused by Staphylococcus warneri. 


\section{Case presentation}

A female patient of Greek origin, 36years old, came in for outpatient urine culture due to symptoms from the lower urinary tract. The patient had suffered surgery five years ago for spinal fusion, which caused damage to the spinal cord and resulting in the development of neurogenic bladder. Since then she is catheterized daily with disposable catheters and often presented urinary tract infection (UTI) caused by $E$. coli. Urine culture was inoculated onto MacConkey 2 and Blood agar (bioMérieux). The plates were incubated at $37^{\circ} \mathrm{C}$ under aerobic conditions for 24-48h. After a 24-hour incubation period, large, white, non-haemolytic, smooth colonies with concentration $10^{4} \mathrm{cfu} / \mathrm{ml}$ were seen on blood agar, whereas no growth was observed on MacConkey 2. Gram staining revealed the presence of gram (+), catalase $(+)$, coagulase $(-)$ cocci arranged in tetrads. Identification and susceptibility testing were performed by the Vitek 2 system (bioMérieux). Staphylococcus warneri was identified. The strain showed resistance only to beta-lactams.

\section{Discussion}

The frequent use of indwelling devices and implants as well as the increase number of immunocompromised patients have given rise to infections caused by organisms which are otherwise considered to be contaminates. S. warneri belongs to the group of CoNS which also includes the following species isolated from human clinical specimens: S. epidermidis, S. haemolyticus, S. saprophyticus, S. lugdunensis, S. hominis, S. simulans, S. capitis, S. auricularis, S. cohnii, S. caprae and S. Pasteur.They colonize the skin and mucosa of human and can cause severe infections, mainly in hospitalized patients. They are also common contaminants of cultures, and they are able to produce biofilms on the surface of various materials, some of them of medical importance [2].
Staphylococcus warneri has been found to be responsible for catheter-related bacteremia, native and prosthetic valve endocarditis (PVE), hematogenous vertebral osteomyelitis, ventriculoperitoneal shunt-associated meningitis, spondylodiscitis, septic arthritis, prostatitis and meningitis in a patient with Strongyloides stercoralis [3-5]. According to the literature, the cases of urinary tract infections caused by $S$. warneriare extremely rare.

Our patient was a healthy adult, except that she was catheterised daily in order to void her urine bladder. This fact confirms the association between indwelling devices and CoNS reported in the literature [2, $6,7,9]$

In our case the isolate was susceptible to quinolones, nitrofurantoin, sulfamethoxazole/trimethoprim, linezolid, aminoglycosides, teicoplanin and vancomycin but resistant to beta-lactams. These findings correlate with the results of the research of Marrie and Kwan [6], who tested the susceptibility of 8 strains S.warneri from urine specimen. The isolates were resistant to ampicillin and cephalexin and susceptible to nitrofurantoin and nalidic acid similarly to our. In another multicenter study that was conducted in 12 hospitals in China, urine samples were collected from 356 symptomatic patients treated for acute uncomplicated cystitis, recurrent UTI or complicated UTI. The most frequently identified Gram-positive pathogens were Stahylococcus coagulase negative spp. (17.1\%). Sensitivity rates were $38.5 \%$ to oxacillin, 50\% for levofloxacin, and 100\% for nitrofurantoin [7]. In a recent study (8) a total of 185 clinically- significant CoNS isolates were collected in a department of microbiology from various samples as follows: 85 were from blood culture, 36 from urine samples, 29 from pus, 14 from vaginal swab and 21 from miscellaneous group (drains, catheter, and fluids). Result of antibiotic susceptibility testing showed $100 \%$ sensitivity to vancomycin. Sensitivity to other antibiotic were as follow: amikacin 61.6\%, ciprofloaxcin $35.1 \%$, nalidixic acid $35.1 \%$, gentamicin $33.5 \%$, cefotaxime $31.8 \%$, cefepime $33.5 \%$, co- 
trimaxozocle $26.4 \%$, erythromycin $30.27 \%$, penicillin $G 9.72 \%$ and methicillin (34.5\%). In the cases of CoNS infections reported so far, the microorganisms were found to be at high rates methicillin-resistant, more specifically to ampicillin, amoxy/clav and the cephalosporins, whereas resistance rates to linezolid, vancomycin and teicoplanin was low. Sensitivity to quinolones, sulfamethoxazole/trimethoprim, nitrofurantoin and aminoglycosides vary among studies $[8,9]$. However, a recent paper from Germany has reported increased incidence of linezolid-resistance S. epidermidis isolates from hospitalized patient [10]

\section{Conclusion}

Because CoNS are ubiquitous especially skin colonizers, they are frequently found as contaminants in clinical specimens, a fact that complicates laboratory interpretation of their clinical significance. When these organisms are isolated in routine laboratory in not significant numbers (counts $\leq 10^{5} \mathrm{cfu} / \mathrm{ml}$ ), every effort should be made to substantiate their clinical relevance and antimicrobial susceptibility in each case so that the an effective antibiotic can be used in the treatment. 


\section{References}

1. Jahromi SM, Mure A, Gomez C. UTIs in Patients with Neurogenic Bladder. Curr Urol Rep 2014; 15: 433.

2. Rahman A, Hosaain MA, Mahmud C, Paul SK, Sultana S, Haque $\mathrm{N}$, Kabir MR, Kubayashi N. Species distribution of coagulase negative staphylococci isolated from different clinical specimen. Mymensingh Med J 2012; 21(2):195-9.

3. Garkowski AZajkowska A, Czupryna P, Lebkowski W, Letmanowski M, Gołebicki P, Moniuszko A, Ustymowicz A, Pancewicz S, Zajkowska J. Infectious spondylodiscitis - a case series analysis. Adv Med Sci. 2014 Mar;59(1):57-60.

4. Ferhat A, Nese S, Birgül M, Ali M. Recurrent Staphylococcus warneriiprosthetic valve endocarditis: A case report and review. Ann ClinMicrobiolAntibioc 2011; 10:14.

5. Incani RN, Hernández M, Cortez J, González ME, Salazar YD. Staphylococcuswarneri meningitis in a patient with Strongyloidesstercoralishyperinfectionamd lymphoma: first report of a case. Ren Inst Med Trop Sao Paulo 2010; 52(3): 16970.

6. Marrie TJ, Kwan C. Antimicrobial susceptibility of Staphylococcussaprophyticus and urethral staphylococci. Antimicrob Agents and Chem 1982; 22(3): 395-97.

7. Qiao LD, Chen S, Yang Y, Zhang K, Zheng B, Guo HF et al. Characteristics of urinary tract infection pathogens and their in vitro susceptibility to antimicrobial agents in China: data from a multicenter study. BMJ Open 2013; 3(12): e004152. doi: 10.1136/bmjopen-2013-004152.

8. Sadhvi Parashar. Significance of Coagulase Negative Staphylococci with Special Reference to Species Differentiation and Antibiogram. Indian Medical Gazette 2014; 255-258.

9. Olayemi OA, Nurudeen AO, Louis OE, Frieder S. Antibiotic susceptibility pattern and biofilm formation in coagulase negative staphylococci. J Infect Dev Ctries 2014; 8(12):16431645.

10. Jennifer Bender J, Strommenger B, Steglich $M$, Ortrud Zimmermann $\mathrm{O}$, Ines Fenner Ines et al. Linezolid resistance in clinical isolates of Staphylococcus epidermidis from German hospitals and characterization of two cfr-carrying plasmids. J Antimicrob Chemother 2015; doi:10.1093/jac/dkv025

\section{Comment on this article:}

\section{$f(B)$ in $8+S . P$}

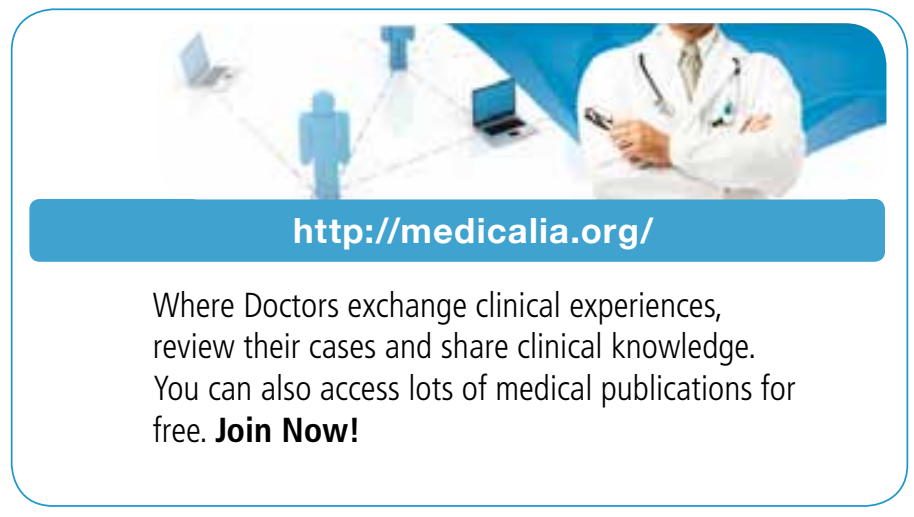

\section{Publish with iMedPub}

http://www.imed.pub

The Journal is an open access peer-reviewed journal that publishes scientific papers about all aspects of antimicrobials. The journal will publish original research articles, reviews, brief reports and case reports dealing with basic and clinical antibacterial agents, antiviral, antiprotozoals, antituberculuous, antifungal and antihelminthes agents. All manuscripts must be prepared in English, and are subject to a rigorous and fair peer-review process. Accepted papers will immediately appear online.

The journal aims to advance the knowledge, attitude and the research of chemotherapy in the Arabic world in cooperation with international, national scientific and public societies as well as research centers with similar aims and objectives.

Submit your manuscript here: www.iajaa.org 